\title{
Role Playing Next Generation 9-1-1: Sensemaking with Social Media in Public-Safety Answering Points
}

\author{
Rob Grace ${ }^{1}$, Andrea Tapia ${ }^{1}$, Jess Kropczynski ${ }^{2}$, Eric Obeysekare ${ }^{1}$, Shane Halse ${ }^{1}$, Julien Coche ${ }^{3}$, Aurélie Montarnal ${ }^{3}$, \\ Mike Beagles ${ }^{4}$, Fred Fonseca ${ }^{1}$ \\ Pennsylvania State University ${ }^{1}$, University of Cincinnati ${ }^{2}$, École des Mines d'Albi-Carmaux ${ }^{3}$, Mission Critical \\ Partners $^{4}$ \\ wrg123@ist.psu.edu, atapia@ist.psu.edu, jess.kropczynski@uc.edu, ero5004@ist.psu.edu, seh297@ist.psu.edu, \\ julien.coche@ orange.fr, aurelie.montarnal@mines-albi.fr, mikebeagles@mcp911.com,ffonseca@ist.psu.edu
}

\begin{abstract}
For over a decade, research has suggested that social media can enhance the situational awareness of emergency responders during a crisis. Rarely, however, do studies examine the sensemaking processes of emergency responders by which situational awareness is achieved. We examine sensemaking in a Public-Safety Answering Point (PSAP) through role plays with 9-1-1 telecommunicators that imagine how social media analysts can contribute to sensemaking processes among 9-1-1 call takers, dispatchers, and emergency responders. These role plays suggest social media can address information gaps that emerge when 9-1-1 callers fail to provide critical information and vice versa, suggesting social media enhances situational awareness only when integrated into sensemaking processes that synthesize information across multiple, incomplete, but complementary data sources. This synthesis, however, requires cooperative information gathering and sharing among call takers, dispatchers, and social media analysts that PSAPs can coordinate using common interpretive frameworks and common information spaces.
\end{abstract}

\section{Shifting Paradigms}

Research continues to intensely examine social media data as a source of situational information during emergencies [26, 39]. Many studies detail the types of situational information posted on social media during crises [31, 35, 39], techniques for collecting and analyzing social media data $[3,8,15,18,24]$ and situational information needs among emergency responders and citizens $[6,12,14,30]$. These studies typically characterize social media data as a rich source for timely and accurate situational information [39]. Surprisingly few studies, however, consider the sensemaking processes of emergency responders in which social media represents but one of many information sources by which an emergency "situation" becomes intelligible [17, 23, 28, 41]. These processes are complex: sensemaking intertwines the (social-)technical processes for collecting, processing, and visualizing information posted on social media, with the social(-technical) workflows of emergency responders who uptake this information to guide decision-making during emergencies.

Two shifts now disrupt sensemaking among emergency responders that stand to re-shape opportunities for situational awareness during emergency situations. First, as multiple citizens can use multiple communications channels to report information during emergencies, emergency reporting becomes increasingly distributed among crowds of citizens using diverse Information and Communications Technologies (ICTs) to directly and indirectly report emergency information to both emergency responders and networks of citizens $[1,5$, 26].

In addition to directly reporting emergencies by, for instance, calling 9-1-1 in the United States, citizens can indirectly report emergencies on social media that reach other citizens but often not emergency services [9]. Consequently, the unique information cycle enabled by social media [35] creates opportunities for sensemaking and situational awareness among citizens themselves, bypassing professional emergency responders and traditional emergency communications infrastructures [27] such as 9-1-1 or the Integrated Public Alert and Warning Systems (IPAWS). Moreover, as witnessed in operations of the "Cajun Navy" during Hurricane Harvey [4], citizens using social media can coordinate an ad hoc emergency response when professional responders prove unresponsive. The paradigm of centralized emergency response- in which one citizen, on one channel, reports an emergency to professional responders- is far from 
dead, but now competes with an alternative paradigm characterized by decentralized information flows and non-professional actors responding to emergencies.

The second shift mirrors distributed reporting, as information work in emergency dispatch becomes increasingly distributed among multiple dispatchers monitoring multiple sources of citizen-reported information. The tasks of answering citizen's emergency reports and dispatching the appropriate emergency response- now performed by 9-1-1 call takers and dispatchers working in Public-Safety Answering Points (PSAPs)- are expanding with Next Generational 9-1-1 systems that allow citizens, in addition to calling 9-1-1 via mobile or landline, to directly report emergencies via text message (SMS) or, in some cases, web-based reporting channels [13]. To account for these channels, emergency dispatch workflows now involve multiple call takers, dispatchers, and new specializations such as the communications analyst, a position tasked with analyzing and synthesizing information reported on social media with information gathered from other data sources, including 9-1-1 calls and radio reports from emergency responders. As a result, in PSAPs located in mid-size and large cities, emergency dispatch becomes an increasingly distributed and cooperative activity performed among multiple specialized officials working in an increasingly data rich environment.

More broadly, the shifts to distributed reporting and dispatch align with visions of the "smart city" that deploy citizen sensing systems (also referred to as social, human, or urban sensing) that crowdsource remote data collection tasks among citizens located in a geographic area [5, 19]. These approaches can involve collecting data produced by smartphone sensors or public data produced by citizens using social media applications such as Twitter and Instagram to, for example, monitor urban mobility patterns [19] or track shifts in public sentiment around major events [29]. The collection and analysis of social media data has received significant attention as a way to support situational awareness among public safety officials managing emergency services [32], with studies examining the use of social media among police officers [22], public information officers [14], and emergency managers [9].

Citizen sensing represents an effort to align distributed models of emergency reporting and dispatch. By incorporating citizen sensing systems, emergency dispatch centers such as PSAPs stand to coordinate the decentralized information flows between crowds of citizens reporting emergencies, requiring help, or coordinating ad-hoc assistance, and emergency services and community organizations positioned to provide aid. However, as earlier described, existing emergency dispatch centers such as PSAPs stand to be significantly re-structured by incorporating citizen sensing systems, multiple data sources, and the distribution and specialization of emergency dispatch personnel.

\subsection{Exploring Next-Generation 9-1-1}

In this paper, we investigate transformations in emergency dispatch by adopting a sensemaking approach to understand future workflows among Next Generation 9-1-1 systems and telecommunicators serving in PSAPs. Specifically, we examine the results of role play activities involving 9-1-1 call takers and dispatchers that explored the future role of a communications analyst using social media to supplement information obtained from 9-1-1 callers and enhance situational information dispatched to emergency responders.

These role plays reveal that PSAPs represent an increasingly data-rich but often information-poor environment and suggest social media can address information gaps that emerge when 9-1-1 callers fail to provide critical information, and vice versa. Consequently, social media can enhance situational awareness only when integrated into sensemaking processes that synthesize information across multiple, incomplete, but complementary data sources. This synthesis, however, requires multiple officials to be aware of information gaps developing around breakdowns in 9-1-1 call taking and initiate distributed processes of information gathering and sharing. We find that PSAPs coordinate this awareness through protocols providing common interpretive frameworks, and sociotechnical infrastructures providing common information spaces among call takers, dispatchers, and future communications analysts cooperating to make sense of multiple sources of crowdsourced data during an emergency.

\section{A Sensemaking Approach}

Situational awareness represents a foundational concept for research exploring information processing and data fusion, human-centered analytics, and decision-making in data and information rich environments. The common definition of situational awareness comes from Endsley [7] who describes a "state of knowledge" concerning the perception of elements in the environment, comprehension of relations among elements, and projection of these elements' statuses in the future. In contrast, Endsley differentiates situational awareness from what she refers to as "situation assessment," the "processes used 
to achieve that state" [7:36]. This study, roughly, concerns the latter, which has elsewhere been referred to as the process of sensemaking $[10,16,40]$.

If situational awareness concerns a mental representation of elements in an environment, dots that must be connected, sensemaking concerns "the skill needed to identify what counts as a dot in the first place" [16:72]. Importantly, sensemaking does not bracket the "situation" as an independent mental model of the world but considers any situation as a situated encounter with the world that unfolds through (often skilled) engagement in goal-directed activities [11]. As a result, to return to the dot analogy, sensemaking concerns understanding what dots matter in a given situation when pursuing a given task.

In contrast to a situational awareness perspective, a sensemaking approach poses different questions [10:2]. These questions are guided by the three, interconnected aspects of sensemaking.

First, breakdowns in perceived activity prompt sensemaking. As Weick et al. observes:

Explicit efforts at sensemaking tend to occur when the current state of the world is perceived to be different from the expected state of the world... To make sense of the disruption, people look first for reasons that will enable them to resume the interrupted activity and stay in action. These "reasons" are pulled from frameworks such as institutional constraints, organizational premises, plans, expectations, acceptable justifications, and traditions inherited from predecessors. [40:409]

Breakdowns involve contrast between the expected and encountered course of an activity. Importantly, the expected course of an activity implies an interpretive background or framework that shapes our experience of the activity and against which interruptions can be experienced [11]. Two important consequences emerge: without an effective basis for comparison and evaluation (i.e. interpretive framework), more information does not necessarily contribute to better situational awareness (i.e. additional information will not prompt breakdowns and initiate sensemaking), and automated systems for data fusion and decisionsupport may obscure breakdowns that otherwise might appear to human analysts as contrasts between the expected and unexpected among data or data sources [16:72].

Whereas a situational awareness perspective begins with an objective environment of "elements" assumed to be equally noticeable to a decision-maker, a sensemaking perspective begins with occasions of breakdown that occur when the encountered and expected course of a task differs and, as a result, elements of the environment become noticeable to the decision-maker who then reinterprets the situation to resume the task [10:2]. We therefore ask: what occasions breakdowns in 9-1-1 call taking and dispatch, and how can communications analysts working with social media data contribute to the sensemaking efforts they invite?

Second, sensemaking efforts, in turn, draw on interpretive frameworks to understand what matters in a situation. As Weick et al. [40] describe, sensemaking efforts initiated by breakdowns in activity draw on interpretive frameworks in the form of social or institutional norms, past experiences, and protocols. Such protocols reveal what matters in a situation and guide the resumption of the interrupted activity. Thus, when encountering roadway congestion, for example, drivers make use of known detours, prior experiences with area traffic, and the legal framework of driving rules to resume travel to a destination. Whether the median is wide enough to bypass the congestion, for instance, does not figure as an important or noticeable element of the environment for many drivers (but may for some in different driving environments).

While a situational awareness perspective assesses information that went unnoticed or unknown by a decision-maker that would have prevented or solved a problem, a sensemaking approach seeks to understand the frameworks in which the situation was intelligible to the decision maker at the time, and "help the decision maker understand what matters, see relationships... [and] decompose complex information into coherent chunks" [10:2]. We therefore ask: what interpretive frameworks do 9-1-1 telecommunicators draw on to identify situational information, and how can they guide the analysis of social media data?

Third, sensemaking always remains a situated activity, conditioned by local arrangements of human and technical resources for action [34]. In this sense, the interpretive frameworks that guide sensemaking become deployed in the relationship between a decision-maker and the environment, such that understanding what matters emerges through situated interactions with people and things. To refer to the prior traffic example, taking a detour might involve use of a smartphone and services such as Google Maps or Waze, or calling a friend for directions: available resources sustaining inquiry. As a result, while a situational awareness perspective focuses on information inputs, what was or was not available, a sensemaking perspective attempts to understand the processes of interaction between decision-makers and sociotechnical infrastructures that shape the availability of information. We therefore ask: what human and technical infrastructures sustain the sensemaking efforts of 9-1-1 telecommunicators, and how can communications analysts draw on and contribute to these? 


\section{Role Play Methods}

This study reports findings from a design workshop conducted at a PSAP responsible for 9-1-1 call taking and emergency dispatch in an urban, highly-populated county in the United States. The workshop was organized to explore how a communications analyst working with social media could support 9-1-1 call taking and dispatch, and the associated design requirements for awareness-support tools that would enable the analyst in this work.

The workshop involved interviews with PSAP administrative (director, deputy director, and operations manager), telecommunications (floor supervisors, call takers, and dispatchers), and information technology staff (IT manager, CAD supervisor, and CAD technicians), as well as approximately twenty hours of combined observation. During observation periods, the authors more closely examined the workflows of 9-1-1 call takers, dispatchers, and resource managers, and their interactions with Computer-Aided Dispatch (CAD), GIS, radio, and associated systems used in emergency dispatch and response.

The workshop particularly focused on role-play activities in which six 9-1-1 telecommunicators volunteered to participate. Role playing involves a group of people who act out roles in a constructed scene [21, 37]. Widely used in user-centered design, role playing allows researchers to observe plausible interactions among participants, typically end-users and domain-experts, as they develop within hypothetical situations [21]. Role play is especially useful for understanding activity in emergency situations that are difficult to directly observe and inappropriate for research activities [38].

During the workshop, the authors specified the roles- caller, call taker, dispatcher, and emergency responder, as well as citizen bystander and communications analyst- but the scenes were left intentionally semi-scripted so that the telecommunicators could draw on their training and past experiences to construct situations that were both realistic and critical from a design perspective. Prompts included suggesting scenarios that the PSAP had encountered in the past, for example, an active shooter in a local mall.

Qualitative analysis was performed on transcribed role-play sessions and post role-play debriefing sessions. We performed content analysis using open coding strategies as suggested by Strauss [36] to develop a coding schema. Themes and concepts relating to sensemaking breakdowns, interpretive frameworks, and sociotechnical infrastructures were identified, discussed, and refined iteratively among researchers. For each role play, we analyzed whether breakdowns in sensemaking occurred and utilized information gathered from post role-play debriefing sessions to explore the occasions for each breakdown we observed.

\section{Analysis}

We focus on one role-play scenario to illustrate sensemaking in emergency dispatch- to include how social media data can contribute to this process- and examine the aspects of sensemaking that were evoked throughout the role-play sessions.

This role play witnessed 1) a 9-1-1 call break down when the caller did not provide requested information; 2) successive breakdowns for dispatchers and responders who rely on this information; 3) sensemaking among multiple officials who engage multiple data sources to address the resultant information gap; 4) the communications analyst using social media data to provide the needed information that; as a result, 5) allowed the emergency response to resume.

We discuss each stage of the role play based around selected excerpts and giving special attention to the relationships among breakdowns, interpretive frameworks, and infrastructures through which we examine the sensemaking process.

\section{1. "The caller is unresponsive at this time"}

Two primary breakdowns emerge during the role play. The first develops immediately after the call taker answers a 9-1-1 call:

Call Taker: 9-1-1, what is the address of your emergency?

Caller: Girl, you need to get here now and quit playin. You need to get them cops down here now girl!

Call Taker: Ma'am, what is the address of the emergency?

Caller: Man, I know you can see me on the phone.

Call Taker: Ok ma'am, I need the address of the emergency.

Caller: Oh my god, I'm at North Romney.

Call Taker: Ok, do you know the address on North Romney?

Caller: 32B North Romney.

PSAPs set different targets for obtaining the location and chief complaint from callers. In the scenario, the call taker asks four times before the caller describes the location with enough precision for the call taker to 
dispatch a response. Without a specific location, call takers cannot process the call for dispatch: "obviously we can't respond if we don't know where" (P4).

The role play continues as the caller reluctantly answers the call takers' questions:

Call Taker: Thank you, tell me exactly what happened.

Caller: Girl, there's about six people here ganging up on this little black bitch here. You need to come get her...

Call Taker: What's the phone number you are calling from?

Caller: Oh my god! I know you can see my phone number on that screen girl...

Call Taker: Does anyone have any weapons?

Caller: I... don't know.

Call Taker: Ok, how many people are involved?

Caller: Man, there's a bunch. There's a bunch...

Call Taker: Do you know an approximate age range?

Caller: Man, they all from Bayside High School. Why you playin?...

Call Taker: By not answering these questions you may put you and responders at risk. Please allow me to help you by answering these questions... [role play shifts]

The role play- introduced as "a common one for us down here" (P1)- quickly demonstrated what the call takers often experience: uncooperative callers who fail to provide or simply do not know the information requested. "When you are talking to a caller, 90\% of our job they aren't listening, they're not answering the questions" lamented one call taker during our followup discussion (P3). Throughout the workshop, 9-1-1 telecommunicators described a data rich environmentthis PSAP processes over 3000 calls per day- and an information poor environment- callers often prove uncooperative, unreliable, or simply ignorant of events occurring around them. "Probably better," replied the call taker when later asked about the quality of information reported on social media, "because all I got is a screaming person on the line."

As breakdowns occur when a contrast develops between the encountered and expected course of an activity, 9-1-1 call taking breaks down when uncooperative callers interrupt the standardized series of question and answer that allow call takers to obtain the information required for dispatch to emergency responders. Call-taking sequences are structured around two standardized protocols that allow call takers to efficiently question callers and sequentially enter priority information into CAD. The "Six W's"Where, What, Weapons, Who, When, and Whyprovide call takers with a heuristic for questioning callers and entering only relevant information for each call. Second, ProQA, an expert system integrated into CAD, provides call takers with emergency-specific question scripts and caller instructions, standardized text entry forms (i.e. call notes). ProQA also assists call takers in determining the chief complaint code, the emergency classification that determines the type and level of emergency, and, in turn, the police, fire, or EMS resources that will be dispatched. Software such as ProQA, extend the "Six W's" by walking call takers through hundreds of standardized protocols for specific law enforcement, medical, and fire situations.

These protocols tacitly and explicitly determine what information matters for call takers during each 91-1 call: "Those are the most import things we need to know, everything else can, honestly, be thrown in the trash, because that's what we need to know" (P4). As only priority information will be entered CAD- where, what, weapons, etc.- everything else a caller may say during a call will be, in a sense, disposed of by the call taker. Protocols, as interpretive frameworks, shape information gathering and filtering.

The role play next shifts to the dispatcher who, in reality, would be dispatching information to responders at the same time as the call taker is on phone with the caller. The dispatcher receives notice of the 9-1-1 call via CAD as soon as the call taker enters the call (after establishing the "where" and "what") and will dispatch information to emergency responders throughout the call by reading the "call note" updates the call taker continuously enters into CAD while speaking with the caller. Now joining the role play, the dispatcher begins using the (imaginary) radio to communicate with police officers on patrol:

Dispatcher: [via radio] 32B N. Romney Street, $32 B N$. Romney Street, reference to an active disturbance, multiple students, physical, units in route acknowledge

Responder: [via radio] 513 Newark copy... 513

Newark to dispatch, copy?

Dispatcher: Go ahead.

Responder: Does anyone have any weapons?

Dispatcher: Standby... unknown at this time. Call

taker's gathering additional information. Responder: 513 Newark copy.

Here the responder, a police officer in the scenario, easily enters the cooperative arrangement that forms among caller, call taker, and dispatcher, as the officer requests information- the third "w" of the Six W's- in line with the same protocols for information gathering that are already guiding the work of the call taker on the phone with the caller, and the dispatcher who waits for this information to be entered in CAD. Against this common interpretive background, all the officials 
involved recognize this absence of information- silence on the radio, a missing call note- as an information gap that initiates distributed and cooperative sensemaking: each official in his or her own capacity attempts to fill in information about on-scene weapons.

\section{2. "Don't forget to press the period"}

The second breakdown emerges as the dispatcher waits for the call taker to forward call notes regarding possible weapons at the scene of the fight. At this moment, however, the dispatcher interrupts the role play to joke that the call takers' notes might not have "dropped":

P5 (Dispatcher): [Aside to call taker] Wait, don't forget to press the period (laughter among telecommunicators)... There is a flaw in our system right now...

P2 (Call Taker): [Explaining to authors] When we're typing in stuff it's not showing updropping for them [dispatchers], so we have to hit a period and hit enter. So that it drops.

P5 (Dispatcher): And as dispatchers we recently learned that we can also do that, so you will see like sixteen periods.

P3: I have learned if you just hit space and do it, it will drop, and it doesn't put all the annoying periods.

Communication between call takers and dispatchers takes place through "call notes" in CAD, a shared text log among telecommunicators, in which call takers can enter priority information (Six W's) obtained from callers, and dispatchers can enter questions or updates obtained from responders with whom they communicate by radio. When a telecommunicator enters or "drops" information into call notes, that information will automatically update on other telecommunicators' CAD interfaces. If, for example, the call taker cannot enter call information (i.e. if the caller is uncooperative) or the call notes do not drop (i.e. if they do not automatically update on the dispatchers' CAD) then information will not reach the dispatcher and, in turn, the police officer with whom she communicates via radio.

Though the telecommunicators laugh, the failure of call taker information to drop to dispatchers, and vice versa, represents a serious problem: communication breaks down between people requesting and offering help. The aside reveals the technical infrastructure that sustains sensemaking in the PSAP: when call notes fail to drop, they occasion breakdowns in CAD as a common information space [2, 41]. As such, CAD supports awareness of protocol-selected information dropped into the space among telecommunicators and responders to whom this situational information is dispatched. As infrastructure, telecommunicators interact with CAD to gather and share information to sustain call taking and dispatch, respectively. At the same time, they re-construct and re-align this space with each call as they draw on different infrastructures and data sources- radio, telephone, and GIS systems- to search for and enter information to address information gaps that emerge in the common information spaces of CAD.

\section{3. "We out here in Bayside"}

The role play shifts again. Two telecommunicators enter the role play as citizen bystanders using social media. The first describes her likely actions: "Most of the tweets you are going to get in that situation are... going to be other high school students. So, I would have tweeted a picture with \#BurkeHigh and \#Fight, and let it go off from there" (P3). The second took a different approach:

I did live video and commentary, Facebook Live... [begins commentary] "We out here in Bayside, Bangin. Ya'll we need the police, they're always in here messin with us but they ain't here now." And it's just live of people fighting each other. (P4)

These social media posts imagine information that a future communications analyst could use to address gaps emerging in 9-1-1 caller information.

However, when the communications analyst attempts to join the role play and contribute to the sensemaking process currently underway, the telecommunicators pause to quickly coordinate how this could work:

P3: [The comm. analyst] had better be putting in information that says...

P6: (comm. analyst): Wait, where are we? You just said, "no known weapon at this time." And [the Dispatcher] has relayed that to [the Responder] already?

P1: Correct, you're relaying to [the dispatcher]. What you've done is opened up our call, and you're putting [call notes into CAD]... Your initials will be next to [the entered call notes], and [the dispatcher] is reading the call as things are dropping and [the dispatcher] is going to go back over the radio.

P5: (dispatcher): I have the call here [sketching CAD interface with her hands] and we have a notification panel across the top, so you can either drop a note in [call notes] or send a notification [which appears on the top of her screen].

P1: So, what did you put in the notes? 
The telecommunicators position the new analyst vis-avis the same infrastructures that enable 9-1-1 call taking and dispatch. Just as a call taker now uses CAD to open a call and enter call notes while questioning a 9-1-1 caller using protocols to gather and filter information, the analyst will simultaneously open CAD and enter call notes while query social media data using the same protocols. To know what matters in a time-critical situation then, the analyst will draw on standard protocols such as the Six W's:

P5: If you have multiple [data sources], [the comm. analyst is] going to have to sit there and be able to sift through all that information, it's not like he's going to be able to see everything at once. He's going to be like alright, now I got that Facebook Live video, I've got these Twitter posts coming up, and now somebody just posted something on Instagram.

P2: But that's more when you just put notes in the call "Live feed showing handgun present."

P1: I would also assume that we would be just trying to get something particular if he is involved... maybe he [comm. analyst] is not trying to get twenty questions, maybe he is just trying to get one...

And at this moment in the role play, that one piece of information regards weapons. Such protocols, as interpretive frameworks, make breakdowns apparent, revealing what information is missing (e.g., are there weapons?), guide information gathering (e.g. are there indications of weapons on social media?), and sharing (e.g. is information on weapons already available in CAD?) to enable distributed, cooperative sensemaking among multiple officials working across multiple data sources to synthesize information that address common situational awareness needs.

The role play then kicks off again, seamlessly:

Comm. Analyst: [via CAD] Per live feed, weapon on scene, handgun.

Dispatcher: [via radio] Dispatched units, updated information: We have a live feed showing at this time that there are multiple subjects approximately [one handgun] can be viewed at this time.

Comm. Analyst: [via CAD] Update: shots fired.

Dispatcher: [via radio] All units be advised shots fired 32 N. Romney, 32 N. Romney, shots fired.

Responder: [via radio] 513 Newark copy. Dispatch, do we know who if anyone is injured?

Dispatcher: [via Radio] Unknown at this time. We started EMS en route, they will be staging...

Caller: Whooooo, Whooooo, Whoooo, Oh my god! Somebody shot Kiki! Somebody shot Kiki!
As the telecommunicators first explained, by using CAD to enter call notes during a developing emergency the analyst, like a call taker, can contribute to the distributed sensemaking process underway among the caller, call taker, dispatcher, and responder carrying out the emergency response.

\section{Discussion}

To understand these processes, we focus on three interconnected aspects of sensemaking: breakdown, interpretive frameworks, and sociotechnical infrastructure. From the perspective of each aspect we present our findings regarding the integration of communications analysts working with social media data in Public-Safety Answering Points (PSAPs), and implications for research examining social media data as a source of situational information that can contribute to emergency response.

\subsection{Local Breakdowns Invite Sensemaking}

Breakdowns occur when 9-1-1 call takers and dispatchers encounter missing information that interrupts the course of their work. When a 9-1-1 caller proves unresponsive and the scripted question and answer dialogue breaks down, or when entered call notes fail to "drop," call takers and dispatchers encounter information gaps that occasion breakdowns in PSAP workflows. As PSAPs rely almost exclusively on 9-1-1 calls for situational information, information gaps resulting from breakdowns in call taking occasion breakdowns for dispatchers, and, in turn, emergency responders. The tendency for successive local breakdowns in activity identify emergency dispatch and response as inherently distributed and mutuallydependent activities. That is, they involve cooperation and require coordination [33].

However, as the role play demonstrates, local breakdowns can be overcome when distributed officials become aware of the information gap and can draw on alternative information sources to share insights that allow response activities to resume. During the role play, the communications analyst using social media data was able to provide unique information about on-scene weapons that, in turn, allowed the dispatcher to resume her information updates to the emergency responder. Here the 9-1-1 telecommunicators turned to social media for select situational information that was unavailable when relying on information provided by the 9-1-1 caller alone. Critically, the communications analyst was able to draw on interpretive frameworks and sociotechnical infrastructures that conditioned his awareness of the 
information gap, and guided his (imaginary) analysis of social media data and selection of situational updates entered into CAD.

More generally, we find emergency dispatch to involve multiple, incomplete information sources (i.e. 9-1-1 callers) that, individually, often fail to support the information needs of emergency dispatchers and responders. This study suggests, then, that in a datarich environment the availability of situational information on social media is distinct from the utility of that information. As emergency responders rely on multiple data sources, the extent to which social media data can enhance situational awareness depends on the information content obtainable on social media and the extent to which social media data can be integrated into distributed sensemaking processes that coordinate the synthesis of unique information across these data sources.

Our findings therefore contribute to theory surrounding social media and situational awareness by showing that social media content does not, ipso facto, enhance situational awareness in emergency response unless coordinated within the distributed sensemaking processes of emergency responders. Social media cannot be simply "pumped in" to officials but must be coordinated within existing workflows in which it provides incomplete information only in relation to other incomplete information sources. Situational awareness is, therefore, the achievement of domaindependent processes that coordinate the synthesis of information across multiple, incomplete, but complementary data sources to meet unfolding information requirements during an emergency. As a result, opportunities to use social media data to enhance situational awareness require aligning the "inputs" of social media with coordination mechanisms that organize this distributed sensemaking processes.

\subsection{Protocol as Interpretive Framework}

The role play demonstrated how protocols such as the Six W's and ProQA serve as interpretive frameworks during sensemaking processes: showing officials what information matters during an emergency and coordinating information gathering, filtering, and sharing among multiple officials working with multiple data sources. Such protocols are domaindependent and are critical in enabling the cooperative sensemaking processes that support emergency responders' situational awareness.

Studies that seek to understand what types of situational information is available on social media during an emergency using qualitative coding [25, 31, 39], and those that use qualitatively-coded datasets to develop machine learning classifiers to filter situational information posted on social media $[15,18]$, stand to be improved by adopting the criteria for situational information explicit or implicit to the domain-specific protocols of emergency response practitioners.

While machine learning approaches have attempted to identify tweets related to an event lacking common keywords or hashtags [18] or explicit location information [20], understanding domain-specific protocols can help refine classifiers to more accurately filter social media data and identify information that supports emergency responders' sensemaking and situational awareness.

As described by telecommunicators during the workshop, PSAPs typically gather information pertaining to the Six W's, often using the scripted questions provided by ProQA that to obtain information required by emergency responders. Tools supporting future communications analysts will be similarly required to assist analysts by, for example, pre-filtering and visualizing social media data in ways that align with domain-dependent information requirements. Studies that evoke the tacit and explicit protocols of these domains can provide criteria for qualitatively coding social media datasets that, in turn, can inform the development of automated classification methods.

\subsection{Sensemaking Infrastructures}

Lastly, sensemaking infrastructures consist of human and artifactual (e.g. information systems) resources for action [34] that sustain inquiry during sensemaking processes. In the context of the PSAP, we find three intertwined infrastructures: the protocols shared among call takers, dispatchers, and responders, the distributed communication technologies and data sources these officials engage, and the Computer-Aided Dispatch (CAD) system that provides a common information space through which officials can access and share information. Officials draw on and interact with these infrastructures to reveal information gaps associated with data sources (e.g. 9-1-1 callers) and cooperatively gather and share information to address these gaps.

As the role plays suggest these infrastructures- as resources for action- are mutually constituted: CAD, as a common information space, requires officials to enter and share information obtained from different communications channels and data sources (telephone, radio, social media). However, the information officials enter and share is that which addresses information gaps recognized on CAD. As described, protocols shared among officials- as interpretive frameworks- serve as infrastructures and coordination mechanisms that facilitate sensemaking processes by allowing officials to recognize and address common 
information gaps appearing on CAD. We find, then, that prospective communications analysts should be prior telecommunicators or receive the same training in protocols guiding 9-1-1 call taking and dispatch. Furthermore, communications analysts will require the same CAD workstation as telecommunicators, in addition to social media-specific tools that can collect, process, and visualize social media data, so that they can integrate protocol-selected information obtained from social media data into the common information spaces that enable sensemaking processes in the PSAP.

\section{Conclusion}

Through role-play activities with 9-1-1 call takers and dispatchers we illustrate how future communications analysts working with social media data can contribute to processes of sensemaking in PSAPs. By attending to aspects of sensemaking- breakdowns, interpretive frameworks, and sociotechnical infrastructures- this study provides insight into the coordination of sensemaking processes that can enhance situational awareness in near-future environments of distributed emergency reporting, dispatch, and response.

\section{Acknowledgements}

We thank the Charleston County Consolidated 9-1-1 Center and Mission Critical Partners without whom this work would not be possible. This research is supported by the National Science Foundation under Grant No. 541155 and No. 741370.

\section{References}

[1] Ajao, O., J. Hong, and W. Liu, “A survey of location inference techniques on Twitter", Journal of Information Science 41(6), 2015.

[2] Bannon, L., and S. Bødker, "Constructing Common Information Spaces", In Proceedings of the Fifth European Conference on Computer Supported Cooperative Work. Springer Netherlands, Dordrecht, 1997, 81-96.

[3] Bruns, A., and Y.E. Liang, "Tools and methods for capturing Twitter data during natural disasters", First Monday 17(4), 2012.

[4] CajunNavy, "If you or someone you know needs help [Facebook update]", 2017.

https://www.facebook.com/LaCajunNavy/

[5] Cuff, D., M. Hansen, and J. Kang, "Urban sensing", Communications of the ACM 51(3), 2008, pp. 24-33.

[6] Dailey, D., and K. Starbird, "Social Media Seamsters:
Stitching Platforms \&amp; Audiences into Local Crisis Infrastructure", Computer Supported Cooperative Work (CSCW), (2017).

[7] Endsley, M.R., "Toward a Theory of Situation Awareness in Dynamic Systems", Human Factors: The Journal of the Human Factors and Ergonomics Society 37(1), 1995, pp. 3264.

[8] Grace, R., J. Kropczynski, S. Pezanowski, S. Halse, P. Umar, and A. Tapia, "Social triangulation: A new method to identify local citizens using social media and their local information curation behaviors", Proceedings of the 14th ISCRAM Conference 2017-May, 2017, pp. 902-915.

[9] Grace, R., J. Kropczynski, and A. Tapia, “Community coordination: Aligning social media use in community emergency management", Proceedings of the 15th ISCRAM Conference, (2018).

[10] Greitzer, F.L., A. Schur, M. Paget, and R.T. Guttromson, "A sensemaking perspective on situation awareness in power grid operations", 2008 IEEE Power and Energy Society Meeting, IEEE (2008), 1-6.

[11] Heidegger, M., Being and Time, SUNY Press, Albany, 2010 .

[12] Hiltz, S.R., and L. Plotnick, "Barriers to Use of Social Media by Emergency Managers", Journal of Homeland Security and Emergency Management 13(2), 2016, pp. 6-13.

[13] Holland, L., "Charleston County public can now connect with 911 via web - WCBD”, WCBD News 2, 2018. http://www.counton2.com/news/local-news/charlestoncounty-public-can-now-connect-with-911-viaweb/1128896475

[14] Hughes, A., and R. Shah, "Designing an Application for Social Media Needs in Emergency Public Information Work", Proceedings of the 19th International Conference on Supporting Group Work - GROUP '16, ACM Press (2016), 399-408.

[15] Imran, M., C. Castillo, F. Diaz, and S. Vieweg, "Processing Social Media Messages in Mass Emergency", ACM Computing Surveys 47(4), 2015, pp. 1-38.

[16] Klein, G., B. Moon, and R.R. Hoffman, "Making Sense of Sensemaking 1: Alternative Perspectives", IEEE Intelligent Systems 21(4), 2006, pp. 70-73.

[17] Landgren, J., "Supporting fire crew sensemaking enroute to incidents", International Journal of Emergency Management 2(3), 2005, pp. 176.

[18] Li, H., D. Caragea, C. Caragea, and N. Herndon, "Disaster response aided by tweet classification with a domain adaptation approach", Journal of Contingencies and Crisis Management 26(1), 2018, pp. 16-27. 
[19] Liu, Y., X. Liu, S. Gao, et al., "Social Sensing: A New Approach to Understanding Our Socioeconomic Environments", Annals of the Association of American Geographers 105(3), 2015, pp. 512-530.

[20] MacEachren, A.M., A. Jaiswal, A.C. Robinson, et al., "SensePlace2: GeoTwitter analytics support for situational awareness", 2011 IEEE Conference on Visual Analytics Science and Technology (VAST), IEEE (2011), 181-190.

[21] Medler, B., and B. Magerko, "The implications of improvisational acting and role-playing on design methodologies", Proceedings of the 28th international conference on Human factors in computing systems - CHI '10, ACM Press (2010), 483.

[22] Meijer, A., and M. Thaens, "Social media strategies: Understanding the differences between North American police departments", Government Information Quarterly 30(4), 2013, pp. 343-350.

[23] Normark, M., and Maria, "Sense-making of an emergency call", Proceedings of the second Nordic conference on Human-computer interaction - NordiCHI '02, ACM Press (2002), 81.

[24] Olteanu, A., C. Castillo, F. Diaz, and S. Vieweg, "CrisisLex: A Lexicon for Collecting and Filtering Microblogged Communications in Crises", Proc. of the 8th International Conference on Weblogs and Social Media, 2014, pp. 376.

[25] Olteanu, A., S. Vieweg, and C. Castillo, "What to Expect When the Unexpected Happens", Proceedings of the 18th ACM Conference on Computer Supported Cooperative Work \& Social Computing - CSCW '15, ACM Press (2015), 994-1009.

[26] Palen, L., K.M. Anderson, G. Mark, et al., A vision for technology-mediated support for public participation \& assistance in mass emergencies \& disasters, British Computer Society, 2010.

[27] Palen, L., and S.B. Liu, "Citizen communications in crisis", Proceedings of the SIGCHI conference on Human factors in computing systems - CHI '07, ACM Press (2007), 727.

[28] Paul, S.A., and M.C. Reddy, "Understanding together", Proceedings of the 2010 ACM conference on Computer supported cooperative work - CSCW '10, ACM Press (2010), 321.

[29] Purohit, H., and A. Sheth, "Twitris v3: From Citizen Sensing to Analysis, Coordination and Action", Seventh International AAAI Conference on Weblogs and Social Media, 2013.

[30] Reuter, C., T. Ludwig, M.-A. Kaufhold, and T.
Spielhofer, "Emergency services' attitudes towards social media: A quantitative and qualitative survey across Europe", International Journal of Human-Computer Studies 95(C), 2016, pp. 96-111.

[31] Saleem, H.M., Y. Xu, and D. Ruths, "Novel Situational Information in Mass Emergencies: What does Twitter Provide?", Procedia Engineering 78, 2014, pp. 155-164.

[32] Salfinger, A., S. Girtelschmid, B. Proll, W. Retschitzegger, and W. Schwinger, "Crowd-Sensing Meets Situation Awareness: A Research Roadmap for Crisis Management", 2015 48th Hawaii International Conference on System Sciences, IEEE (2015), 153-162.

[33] Schmidt, K., and C. Simonee, "Coordination mechanisms: Towards a conceptual foundation of CSCW systems design", Computer Supported Cooperative Work (CSCW) 5(2-3), 1996, pp. 155-200.

[34] Star, S.L., and K. Ruhleder, "Steps Toward an Ecology of Infrastructure: Design and Access for Large Information Spaces", Information Systems Research 7(1), 1996, pp. 111134.

[35] Starbird, K., L. Palen, A.L. Hughes, and S. Vieweg, "Chatter on the red what hazards threat reveals about the social life of microblogged information", Proceedings of the 2010 ACM conference on Computer supported cooperative work - CSCW '10, ACM Press (2010), 241.

[36] Strauss, A.L., Qualitative analysis for social scientists, Cambridge University Press, 1987.

[37] Svanaes, D., and G. Seland, "Putting the users center stage", Proceedings of the 2004 conference on Human factors in computing systems - CHI '04, ACM Press (2004), 479-486.

[38] Valkonen, P., and M. Liinasuo, "Role playing with fire fighters", Proceedings of the 6th Nordic Conference on Human-Computer Interaction Extending Boundaries NordiCHI '10, ACM Press (2010), 805.

[39] Vieweg, S., A.L. Hughes, K. Starbird, and L. Palen, "Microblogging during two natural hazards events", Proceedings of the 28th international conference on Human factors in computing systems - CHI '10, ACM Press (2010), 1079.

[40] Weick, K.E., K.M. Sutcliffe, and D. Obstfeld, "Organizing and the Process of Sensemaking", Organization Science 16(4), 2005, pp. 409-421.

[41] Wolbers, J., and K. Boersma, "The Common Operational Picture as Collective Sensemaking", Journal of Contingencies and Crisis Management 21(4), 2013, pp. 186199. 